O.M. MAKHANETS, A.I. KUCHAK, V.I. GUTSUL

Yu. Fed'kovich National University of Chernivtsi

(2, Kotsyubyns'kyi Str., 58012, Chernivtsi, Ukraine; e-mail: ktf@chnu.edu.ua)

PACS 73.21.-b; 78.67.-n; 78.67.Ch; 73.22.-f; $62.23 . \mathrm{Hj}$

\section{SPECTRAL PARAMETERS OF ELECTRON IN A MULTISHELL SEMICONDUCTOR CYLINDRICAL NANOTUBE WITH A DONOR IMPURITY AT ITS AXIS}

\begin{abstract}
The spectral parameters of an electron in the multishell semiconductor cylindrical nanotube with a donor impurity at its axis have been studied in the framework of the effective mass and rectangular potential models, by using the modified Bethe variational method. The electronimpurity binding energies and the oscillator strengths of intra-band optical quantum transitions have been analyzed as functions of the geometrical parameters of a combined nanotube composed of semiconductors $\mathrm{GaAs}$ and $\mathrm{Al}_{0.4} \mathrm{Ga}_{0.6} \mathrm{As}$.

Ke ywords: semiconductor nanotube, donor impurity, binding energy, oscillator strength.
\end{abstract}

\section{Introduction}

Semiconductor nanoheterosystems have been studied both theoretically and experimentally for more than 20 years. Nowadays, experimental capabilities already allow scientists to create ensembles of simple (with one quantum well for the electron) and combined multishell semiconductor nanotubes with different geometrical shapes [1-4].

A possibility of different quasiparticle localizations in multishell nanotubes allows the latter to be used as basic elements in modern nanodevices (tunnel nanodiodes, nanotransistors, nanosensors, and so forth). The efficiency of such nanodevices should considerably exceed that of available devices functioning on the basis of simple quantum wires. In particular, the probable localization of charge carriers in the internal wire substantially reduces the intensity of surface scattering and interaction with other dissipative subsystems. For such nanosystems with the cylindrical or hexagonal symmetry, the theory of exciton and phonon spectra has already been developed, as well as the theory of electron-phonon and exciton-phonon interaction, which agrees well with both experimental results and the general physical reasoning [5-7].

It is clear that the presence of impurities in nanotubes will considerably affect the physical properties of the latter and, in turn, the physical characteristics of nanodevices created on their basis. At present, the

(c) O.M. MAKHANETS, A.I. KUCHAK, V.I. GUTSUL, 2014

ISSN 2071-0194. Ukr. J. Phys. 2014. Vol. 59, No. 8 possibility of the controlled doping of semiconductor nanosystems with impurities is experimentally implemented only for spherical quantum dots obtained by using the methods of colloid chemistry. In particular, the authors of works $[8,9]$ studied the influence of the impurity localization on the intensity of electron quantum transitions in spherical quantum dots created on the basis of semiconductors ZnSe, CdS, and $\mathrm{ZnS}$. It is clear that the rapid progress of experimental technologies dealing with the epitaxial growth of nanosystems will make it also possible to controllably introduce impurities into combined semiconductor nanotubes $\mathrm{GaAs} / \mathrm{Al}_{x} \mathrm{Ga}_{1-x} \mathrm{As}$ [4].

The research of the binding energy of an electron with an impurity in cylindrical quantum wires or simple nanotubes faces the considerable mathematical difficulties associated with the fact that the spherical symmetry of the Coulomb interaction potential between the electron and the impurity and the nonspherical symmetry of the system have to be put in agreement with each other. Therefore, while studying the impurity-renormalized electron spectrum, the authors use the Ritz variational method in the overwhelming majority of works [10-16]. However, this method allows only the ground state of an electron to be described rather exactly and simply. The choice of a variational function for excited states is ambiguous and cumbersome [15]. Much more informative is the method of effective potential, which was used earlier for the theoretical study of the excitonic spectrum in simple and combined semiconductor nanotubes $[7,17]$. 


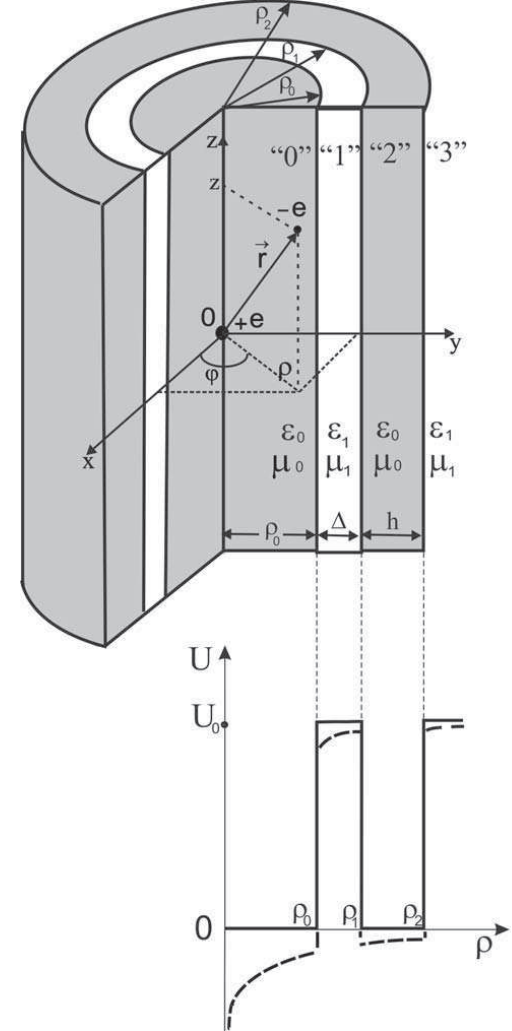

Fig. 1. Geometrical scheme and energy diagram of a multishell nanotube

As far as we know, the issues concerning the interaction of the electron with the donor impurity in a combined multishell semiconductor nanotube have not been studied at all. Therefore, this work aimed at developing one of the possible theoretical approaches to study the energy spectrum of an electron in the combined cylindrical semiconductor nanotube with a donor impurity at its axis. As an example, the dependences of the electron-impurity binding energy and the oscillator strengths of intraband quantum transitions on the geometrical parameters of the nanosystems will be analyzed on the basis of semiconductors GaAs and $\mathrm{Al}_{0.4} \mathrm{Ga}_{0.6} \mathrm{As}$.

\section{Theory of the Energy} Spectrum and Wave Functions of an Electron in the Multishell Cylindrical Semiconductor Nanotube with a Donor Impurity at its Axis

In this paper, we study the combined cylindrical semiconductor nanotube consisting of a quantum wire with radius $\rho_{0}$ (medium 0 , GaAs), a thin semiconductor layer-barrier with thickness $\Delta$ (medium 1 , $\mathrm{Al}_{0.4} \mathrm{Ga}_{0.6} \mathrm{As}$ ), and a nanotube with thickness $h$ (medium 2, GaAs), all embedded in the environment (medium 3, $\mathrm{Al}_{0.4} \mathrm{Ga}_{0.6} \mathrm{As}$ ). The geometrical scheme and the energy diagram of this nanotube are shown in Fig. 1.

For the symmetry reasons, the further calculations are convenient to be carried out in a cylindrical coordinate system $(\rho, \varphi, z)$ with the axis $O z$ directed along the nanotube axis. The donor impurity is located at the coordinate origin and creates an additional attractive Coulomb potential shown by a dashed curve in Fig. 1.

The spectral parameters of the electron interacting with the impurity were calculated in the framework of the effective mass model. Despite its relative simplicity, this model adequately describes the energy spectra of electrons, holes, or excitons in nanosystems with various symmetries. The results obtained in this model completely agree with experimental measurements even for nanosystems a few nanometers in dimensions $[5,7,18,19]$.

Hence, the dielectric permittivities and the effective masses and potential energies of the electron are considered to be known for every region in the combined nanotube,

$$
\begin{aligned}
& \varepsilon(\rho)=\left\{\begin{array}{l}
\varepsilon_{0}, \\
\varepsilon_{1},
\end{array} \quad \mu(\rho)=\left\{\begin{array}{l}
\mu_{0}, \\
\mu_{1},
\end{array}\right.\right. \\
& U(\rho)=\left\{\begin{array}{l}
0, \quad 0 \leq \rho \leq \rho_{0}, \quad \rho_{1} \leq \rho \leq \rho_{2}, \\
U_{0}, \quad \rho_{0} \leq \rho \leq \rho_{1}, \quad \rho>\rho_{2} .
\end{array}\right.
\end{aligned}
$$

In order to find the energy spectrum and the wave functions for the electron interacting with the impurity, we have to solve the stationary Schrödinger equation

$\hat{H}(\rho, \varphi, z) \Psi(\rho, \varphi, z)=E \Psi(\rho, \varphi, z)$

with the Hamiltonian

$\hat{H}(\rho, \varphi, z)=\hat{H}_{0}(\rho, \varphi)-\frac{\hbar^{2}}{2 \mu(\rho)} \frac{\partial^{2}}{\partial z^{2}}+V(\rho, z)$.

Here,

$\hat{H}_{0}(\rho, \varphi)=-\frac{\hbar^{2}}{2} \nabla_{\rho, \varphi} \frac{1}{\mu(\rho)} \nabla_{\rho, \varphi}+U(\rho)$

is the Hamiltonian that describes the electron motion in the plane perpendicular to the nanotube axis when the impurity is absent, the second term on the righthand side of Eq. (3) determines the kinetic energy of 
the electron along the axis $O z$, and

$V(\rho, z)=-\frac{e^{2}}{\varepsilon(\rho) \sqrt{\overline{\rho^{2}+z^{2}}}}$

is the potential energy of interaction between the electron and the impurity.

Note that the Schrödinger equation with Hamiltonian (4) can be solved exactly. Since the corresponding theoretical calculation method was described in our works $[5,6]$ in detail, we omit all analytical calculations and consider that the energy spectrum $E_{n_{\rho} m}^{(0)}$ and the wave functions

$\varphi_{n_{\rho} m}^{(0)}(\rho, \varphi)=R_{n_{\rho} m}(\rho) \exp (i m \varphi)$

of an electron in the impurity-free nanosystem are known. In expression (6), $R_{n_{\rho} m}(\rho)$ are the radial wave functions, which are, in the general case, superpositions of the Bessel and Neumann functions of the integer order; and $n_{\rho}=1,2,3, \ldots$ and $m=0, \pm 1, \pm 2$ are the radial and magnetic quantum numbers, respectively.

The Schrödinger equation (2) with Hamiltonian (3) cannot be solved exactly due to the complicated dependences of the interaction potential energy (5) on the variables $\rho$ and $z$ and the dielectric permittivity on the variable $\rho$. The approximate solution of the problem can be sought as follows.

The data in Table demonstrate that the values of $\varepsilon_{0}$ and $\varepsilon_{1}$ are so close to each other that the combined cylindrical nanotube can be regarded as homogeneous with the constant average dielectric permittivity $\bar{\varepsilon}=\left(\varepsilon_{0}+\varepsilon_{1}\right) / 2$. The potential energy of interaction between the electron and the impurity is averaged, by using the electron wave functions (6),

$V_{n_{\rho} m}(z)=\frac{e^{2}}{\bar{\varepsilon}} \iint \frac{\left|\varphi_{n_{\rho} m}^{(0)}(\rho, \varphi)\right|^{2}}{\sqrt{\rho^{2}+z^{2}}} \rho d \rho d \varphi$.

Note that potential (7) not only describes the Coulomb interaction between the electron and the impurity along the axis $O z$, but also "effectively" takes it into account in the perpendicular plane.

Now, in the internal wire (medium 0) or nanotube (medium 2) volume, Hamiltonian (3) averaged over functions (6) looks like

$\hat{H}(z)=-\frac{\hbar^{2}}{2 \mu_{0}} \frac{\partial^{2}}{\partial z^{2}}-V_{n_{\rho} m}(z)+E_{n_{\rho} m}^{(0)}$.
Surely, the Schrödinger equation with Hamiltonian (8) for the $z$-component cannot be solved exactly. In order to obtain its approximate solution, let us apply the modified Bethe variational method [20]. In Hamiltonian (8), let us add and subtract the potential

$V(z)=-\frac{e^{2}}{\bar{\varepsilon}} \frac{1}{(\beta+|z|)}$

with the variational parameter $\beta$. On the one hand, this potential has the main properties of the effective interaction potential between the electron and the impurity (7); therefore, being summed up with the kinetic energy of the $z$-component, it gives the bound state energy $E_{n_{z}}$. On the other hand, its difference with potential ( 7 ) is a small enough quantity in the sense of perturbation theory,

$\Delta V_{n_{\rho} m}(z)=\frac{e^{2}}{\bar{\varepsilon}}\left(\frac{1}{\beta+|z|}-V_{n_{\rho} m}(z)\right)$.

The Schrödinger equation for the $z$-component,

$\hat{H}_{z} \Psi_{n_{z}}(z)=E_{n_{z}} \Psi_{n_{z}}(z)$,

with the Hamiltonian

$\hat{H}_{z}=-\frac{\hbar^{2}}{2 \mu_{0}} \frac{\partial^{2}}{\partial z^{2}}-\frac{e^{2}}{\bar{\varepsilon}} \frac{1}{(\beta+|z|)}$

can be solved exactly [21], and the corresponding wave function has the form

$\Psi_{n_{z}}(\beta, z)=A \exp (-\chi(z+\beta)) \times$

$\times U\left(-\frac{\nu}{2 \chi} ; 0 ; 2 \chi(z+\beta)\right)$

Here,

$\nu=\frac{2 \mu_{0}}{\hbar^{2}} \frac{e^{2}}{\bar{\varepsilon}}, \quad \chi^{2}=\frac{2 \mu_{0}}{\hbar^{2}} E_{n_{z}}$,

$A$ is the normalizing factor, and $U$ the confluent hypergeometric function.

Parameters of nanosystem components

\begin{tabular}{|l|c|c|c|c|}
\hline & $\mu_{0}$ & $U_{0}, \mathrm{MeV}$ & $\varepsilon$ & $a, \AA$ \\
\hline $\mathrm{GaAs}$ & $0.063 m_{0}$ & 297 & $\begin{array}{l}12.9 \\
11.764\end{array}$ & 5.65 \\
$\mathrm{Al}_{0.4} \mathrm{Ga}_{0.6} \mathrm{As}$ & $0.0962 m_{0}$ & & \\
\hline
\end{tabular}


Since Eq. (11) with Hamiltonian (12) is symmetric with respect to the transformation $z \rightarrow-z$, its solutions must be either even or odd. This requirement brings us to two boundary conditions,

$\left.\frac{\partial \Psi_{n_{z}}(z)}{\partial z}\right|_{z=0}=0, \quad \Psi_{n_{z}}(0)=0$

whence the energy spectrum $E_{n_{z}}(\beta)$ can be determined unambiguously. Now, the electron energy as a function of the variational parameter $\beta$ is given by the expression

$E_{n_{\rho} m n_{z}}(\beta)=E_{n_{\rho} m}^{(0)}+\Delta E_{n_{\rho} m n_{z}}(\beta)$,

and the wave functions are

$\Psi_{n_{\rho} m n_{z}}(\beta, \mathbf{r})=\Psi_{n_{z}}(\beta, z) \varphi_{n_{\rho} m}^{(0)}(\rho, \varphi)=$ $=\left|n_{\rho} m n_{z}\right\rangle$.

The impurity-electron binding energy $\Delta E_{n_{\rho} m n_{z}}(\beta)$ in Eq. (16) consists of the bound state energy $E_{n_{z}}(\beta)$ along the axis $O z$ and the correction $\delta E_{n_{\rho} m n_{z}}(\beta)$,

$\Delta E_{n_{\rho} m n_{z}}(\beta)=E_{n_{z}}(\beta)+\delta E_{n_{\rho} m n_{z}}(\beta)$.

The latter is calculated as a diagonal matrix element of the perturbation operator (10) with wave functions (13),

$\delta E_{n_{\rho} m n_{z}}(\beta)=\frac{e^{2}}{\bar{\varepsilon}}\left\langle n_{z}\left|\left(\frac{1}{\beta+|z|}-V_{n_{\rho} m}(z)\right)\right| n_{z}\right\rangle$.

Having found $\bar{\beta}$ corresponding to the minimum $\Delta E_{n_{\rho} m n_{z}}(\bar{\beta})$ of functional (18), we obtain final expressions for the energy spectrum (16) and the wave functions (17) of an electron in the combined cylindrical semiconductor nanotube with a donor impurity.

The obtained electron wave functions (17) also make it possible to estimate the oscillator strengths for intra-band optical quantum transitions according to the known formula [22, 23]

$F_{n_{\rho} m n_{z}}^{n_{\rho}^{\prime} m^{\prime} n_{z}^{\prime}} \sim\left(E_{n_{\rho}^{\prime} m^{\prime} n_{z}^{\prime}}-E_{n_{\rho} m n_{z}}\right)\left|M_{n_{\rho} m n_{z}}^{n_{\rho}^{\prime} m^{\prime} n_{z}^{\prime}}\right|^{2}$,

where

$M_{n_{\rho} m n_{z}}^{n_{\rho}^{\prime} m^{\prime} n_{z}^{\prime}}=\int \psi_{n_{\rho} m n_{z}}^{*}(\bar{\beta}, \mathbf{r}) e \rho \times$

$\times \cos \varphi \psi_{n_{\rho}^{\prime} m^{\prime} n_{z}^{\prime}}\left(\bar{\beta}^{\prime}, \mathbf{r}\right) d \mathbf{r}$

is the dipole moment of the transition.

Note that, by using the explicit form for the wave functions (6) in formula (21), it is easy to find the corresponding selection rules. According to them, only those transitions between the electron energy levels are permitted, for which the difference between the magnetic quantum numbers equals $\pm 1(\Delta m= \pm 1)$.

\section{Analysis and Discussion of Results}

The calculation of the electron spectrum and the analysis of its properties were carried out numerically for a combined multishell cylindrical nanotube on the basis of semiconductors GaAs and $\mathrm{Al}_{0.4} \mathrm{Ga}_{0.6} \mathrm{As}$ with the material parameters quoted in Table $\left(m_{0}\right.$ is the electron mass in vacuum). In what follows, only those transitions between electron levels are considered, for which $n_{\rho}=n_{z}=1$ and $\Delta m= \pm 1$, because, as researches show, just those transitions dominate. Therefore, the subscripts $n_{\rho}$ and $n_{z}$ are omitted below for convenience.

In Fig. 2, $a$ and $b$, the dependences of the electronimpurity binding energy $\Delta E_{m}$ on the radius $\rho_{0}$ of the internal GaAs wire are depicted for two values of nanotube thickness: $h_{1}=4 \mathrm{~nm}$ (panel $a$ ) and $h_{2}=6.5 \mathrm{~nm}$ (panel $b$ ), and at the fixed layer-barrier thickness $\Delta=4 \mathrm{~nm}$. Just those $\Delta$ - and $h$ - values are typical of experimentally created semiconductor nanotubes [4]. One can see that the electron binding energy nonmonotonously depends on the internal wire radius $\rho_{0}$ in all states, with the corresponding minimum and maximum values. Such a behavior of $\Delta E_{m}$ can easily be understood from simple physical reasonings.

For this purpose, it is expedient to consider the probability density distribution to find the electron in the ground state in the nanosystem for three $\rho_{0^{-}}$ values $-\rho_{0}=0$ (panel $\left.c\right), \rho_{0} \approx 5 a_{\mathrm{GaAs}}($ panel $d)$, and $\rho_{0} \approx 9 a_{\mathrm{GaAs}}($ panel $e$ ) - corresponding to points 1 , 2 , and 3 , respectively, in panel $a$. One can see that, if $\rho_{0}=0($ panel $c)$, the quantum wire is absent, and the electron is localized in the nanotube volume with the thickness $h_{1}=4 \mathrm{~nm}$, being characterized by the binding energy $\Delta E_{1} \approx-14 \mathrm{meV}$ (point 1 in panel $a$ ). In the presence of an internal wire, the absolute value of binding energy first decreases, because the effective distance between the electron and the impurity grows. The minimum value of the binding energy with $\left|\Delta E_{1}\right| \approx 10 \mathrm{meV}$ is reached at $\rho_{0} \approx 5 a_{\mathrm{GaAs}}$ (point 2). As is seen from panel $d$, this is the $\rho_{0}$-value, at which the wave function of the electron starts to penetrate into the volume of the internal wire, the effective distance between the electron and the impurity decreases, and the absolute value of $\Delta E_{1}$ grows with the radius $\rho_{0}$. The maximum value of binding energy, $\left|\Delta E_{1}\right| \approx 21 \mathrm{meV}$, is reached at $\rho_{0} \approx 9 a_{\mathrm{GaAs}}$ (point 3 in panel $a$ ). Panel $e$ demonstrates that, at 

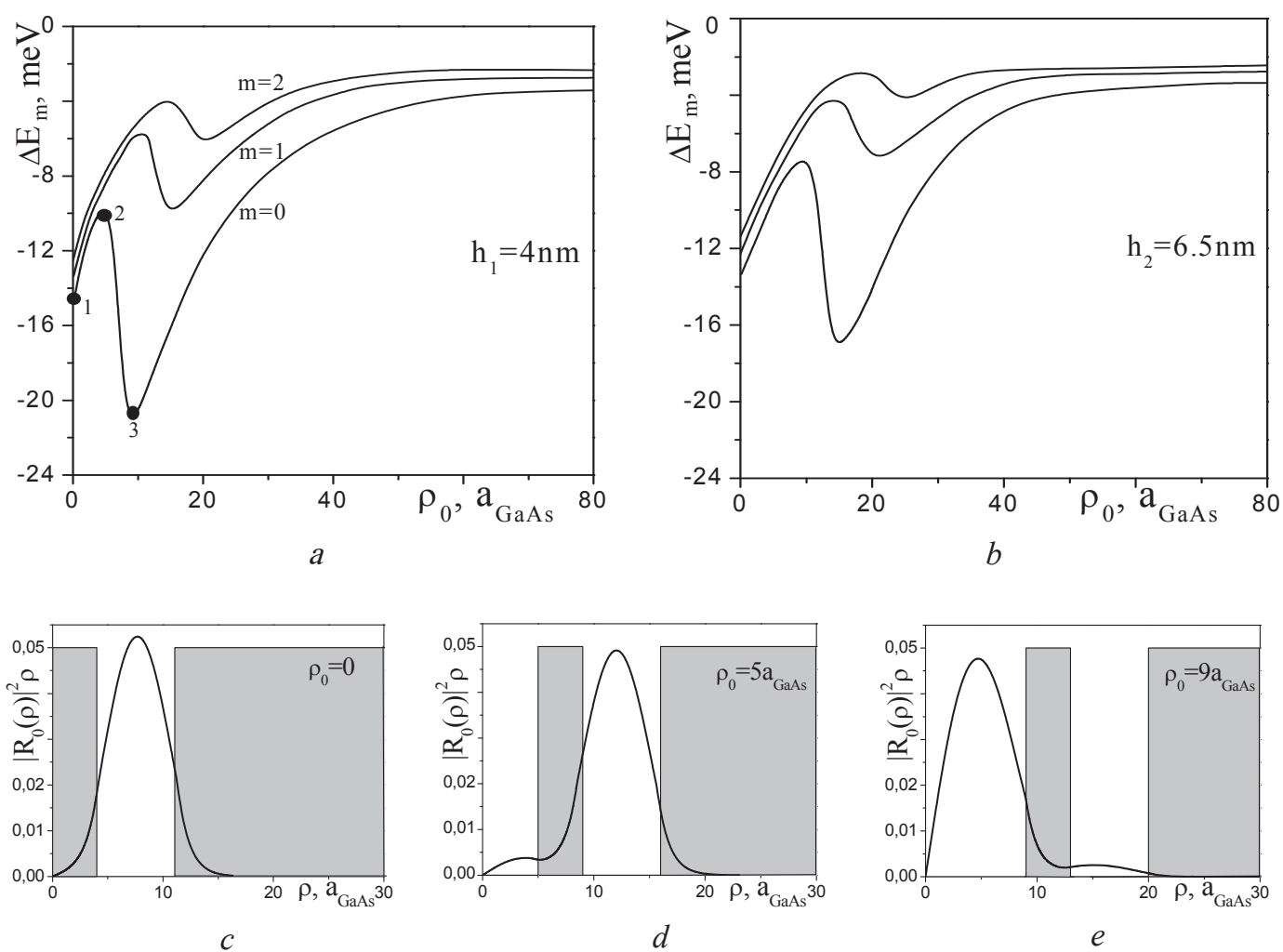

Fig. 2. Dependences of the energy of binding of the electron with the impurity, $\Delta E_{m}$, on the radius of the internal GaAs wire, $\rho_{0}$, for two values of nanotube thickness, $h_{1}=4 \mathrm{~nm}(a)$ and $h_{2}=6.5 \mathrm{~nm}(b)$, and at the fixed layer-barrier thickness $\Delta=4 \mathrm{~nm}$; probability density distributions for the location of the electron in the ground state in the nanosystem with various $\rho_{0}=0(c)$, $5 a_{\mathrm{GaAs}}(d)$, and $\rho_{0}=9 a_{\mathrm{GaAs}}(e)$
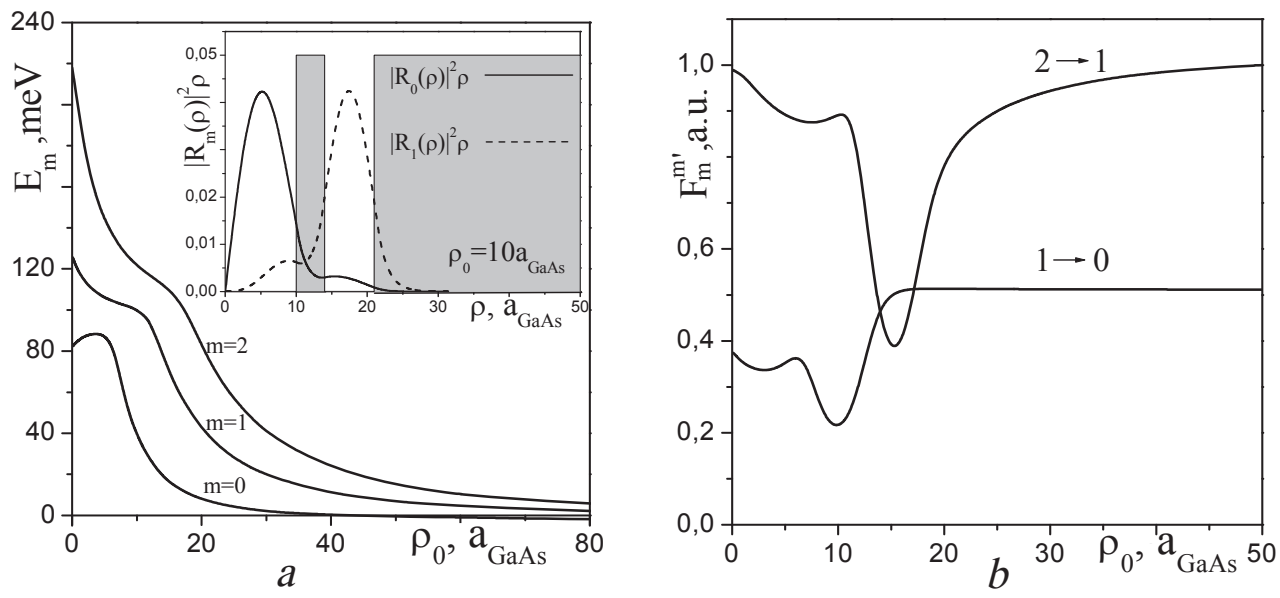

Fig. 3. Dependences of the electron energies $E_{m}(m=0,1,2)(a)$ and the oscillator strengths of intra-band optical quantum transitions, $F_{m}^{m^{\prime}}(1 \rightarrow 0$ and $2 \rightarrow 1),(b)$ on the internal GaAs wire radius $\rho_{0}$ for the fixed layer-barrier $(\Delta=4 \mathrm{~nm})$ and nanotube $(h=4 \mathrm{~nm})$ thicknesses 
this moment, the electron becomes localized inside the internal wire with a probability close to 1 , and the effective distance between the electron and the impurity is minimum. As the internal wire radius increases further, the absolute value of binding energy only decreases and saturates at large enough $\rho$ 's.

In a similar way, the change of the electron localization in the volume of the quantum wire and the nanotube that are coupled with each other by means of tunneling can explain the nonmonotonic dependence of the binding energies of the electron in its excited states. By comparing panels $a$ and $b$, one can see that an increase of the nanotube thickness $h$ gives rise to a shift of all binding energy maxima and minima toward larger internal wire radii, $\rho_{0}$, whereas the distance between them diminishes on the energy scale.

In Fig. 3, the dependences of the electron energies $E_{m}(m=0,1,2)$ (panel $a$ ) and the oscillator strengths of intra-band optical quantum transitions, $F_{m}^{m^{\prime}},(1 \rightarrow 0$ and $2 \rightarrow 1)$ (panel $b$ ) on the internal GaAs wire radius $\rho_{0}$ are plotted for the fixed layerbarrier $(\Delta=4 \mathrm{~nm})$ and nanotube $(h=4 \mathrm{~nm})$ thicknesses. As is seen from panel $a$, the dependence of each electron energy $E_{m}$ on $\rho_{0}$ falls down nonmonotonically, which, as was described above, can be explained by different localizations of the quasiparticle in the combined nanotube. Note also that, owing to a reduction of the binding energy by absolute value in the interval of radii $0 \leq \rho_{0} \leq 5 a_{\mathrm{GaAs}}$, the energy of the electron ground state first increases a little as $\rho_{0}$ grows, reaches a maximum, and only then decreases.

A possibility for the electron in different states to be localized in either the internal wire $\left(\rho_{0}\right)$ or the nanotube $(h)$ volume results in a complicated and nonmonotonic dependence of the oscillator strengths of intra-band quantum transitions on $\rho_{0}$ (Fig. $3, b$ ). In particular, the corresponding dependences demonstrate a minimum of $F_{m}^{m^{\prime}}$ for both types of transitions (in a vicinity of $\rho_{0} \approx 10 a_{\mathrm{GaAs}}$ for transition $1 \rightarrow 0$ ). The minimum in the dependence $F_{0}^{1}\left(\rho_{0}\right)$ at $\rho_{0} \approx 10 a_{\mathrm{GaAs}}$ is a result of the weak overlapping between the wave functions of the electron in the states with $m=0$ and $m=1$, which is well illustrated in the inset of panel $a$. If the internal wire radius is small $\left(\rho_{0}<6 a_{\mathrm{GaAs}}\right)$ or large $\left(\rho_{0}>15 a_{\mathrm{GaAs}}\right)$, the overlapping of the wave functions of the electron in the states with $m=0$ and $m=1$ is substantial, and, therefore, the magnitude of $F_{0}^{1}$ also turns out considerable. The analogous reasoning makes it possible to explain the complicated behavior of the oscillator strength for the electron quantum transition between the states with $m=2$ and $m=1$.

Note also that, as is seen from Fig. 3, b, the oscillator strength $F_{1}^{2}$ of the quantum transition substantially exceeds the oscillator strength $F_{1}^{2}$ within the whole interval of the values of radius $\rho_{0}$, except for a small vicinity of the point $\rho_{0} \approx 16 a_{\mathrm{GaAs}}$.

\section{Conclusions}

A theoretical approach has been proposed to study the spectral parameters of the electron in a combined cylindrical semiconductor nanotube with a donor impurity at its axis in the framework of the effective mass and rectangular potential models. The energies of binding of the electron with the impurity and the oscillator strengths of intra-band quantum transitions were shown to depend nonmonotonically and in a complicated way on the radius of the internal wire $\rho_{0}$, reaching the corresponding minimum and maximum values. Such a behavior of the spectral parameters of the electron is completely governed by a complicated character of the probability density distribution for the location of the quasiparticle interacting with the impurity over the volume of the multishell semiconductor nanotube.

1. P. Mohan, J. Motohisa, and T. Fukui, Appl. Phys. Lett. 88, 013110 (2006).

2. P. Mohan, J. Motohisa, and T. Fukui, Appl. Phys. Lett. 88, 133105 (2006).

3. M. Heigoldt, J. Arbiol, D. Spirkoska et al., J. Mater. Chem. 19, 840 (2009).

4. A. Fontcuberta i Morral, D. Spirkoska, J. Arbiol et al., Small 4, 899 (2008).

5. O.M. Makhanets, N.R. Tsiupak, O.M. Voitsekhivska, Semicond. Phys. Quant. Electr. Optoelectr. 15, 156 (2012).

6. O.M. Makhanets, N.R. Tsiupak, and V.I. Gutsul, Ukr. Fiz. Zh. 57, 1060 (2012).

7. O.M. Makhanets, V.I. Gutsul, N.R. Tsiupak et al., Condens. Matter Phys. 15, 33704 (2012).

8. Y. Yang, O. Chen, A. Angerhofer, and Y. Charles Cao, J. Am. Chem. Soc. 128, 12428 (2006).

9. N. Pradhan, D. Goorskey, J. Thessing, and X. Peng, J. Am. Chem. Soc. 127, 17586 (2005).

10. I.F.I. Mikhail, S.B.A. El Sayed, Superlatt. Microstruct. 55, 198 (2013).

ISSN 2071-0194. Ukr. J. Phys. 2014. Vol. 59, No. 8 
11. S. Aktas, F.K. Boz, and S.S. Dalgic, Physica E 28, 96 (2005).

12. B.Zh. Poghosyan, Nanoscale Res. Lett. 2, 515 (2007).

13. U. Yesilgul, S. Sakiroglu, E. Kasapoglu et al., Superlatt. Microstruct. 48, 106 (2010).

14. N. Porras Montenegro, J. Lopez-Gondar, and L.E. Oliveira, Phys. Rev. B 43, 1824 (1991).

15. I.F.I. Mikhail and S.B.A. El Sayed, Physica E 42, 2307 (2010).

16. F.A.P. Osorio, A.N. Borges, A.A. Caparica, and J.R. Leite, Solid State Commun. 103, 375 (1997).

17. M. Tkach, O. Makhanets, M. Dovganiuk, and O. Voitsekhivska, Physica E 41, 1469 (2009).

18. M.V. Tkach,, V.A. Golovatskyi, O.M. Voitsekhivska et al., Zh. Fiz. Dosl. 4, 342 (2000).

19. G.G. Zegrya, N.V. Tkach, I.V. Boiko, and Yu.A. Seti, Fiz. Tverd. Tela 55, 2067 (2013).

20. H.A. Bethe and E.E. Salpeter, Quantum Mechanics of One- and Two-Electron Atoms (Springer, Berlin, 1957).

21. T. Ogawa and T. Takagahara, Phys. Rev. B 44, 8138 (1991).
22. M. Masale, Physica B 292, 241 (2000).

23. M. Masale, Physica B 291, 256 (2000).

Received 20.02.2014.

Translated from Ukrainian by O.I. Voitenko

О.М. Маханецъ, А.І.Кучак, В.І. Гуцул

СПЕКТРАЛЬНІ ПАРАМЕТРИ

ЕЛЕКТРОНА В БАГАТОШАРОВІЙ

ЦИЛІНДРИЧНІЙ НАПІВПРОВІДНИКОВІЙ

НАНОТРУБЦІ 3 ДОНОРНОЮ ДОМІШКОЮ

НА АКСІАЛЬНІЙ ОСІ

$\mathrm{P}$ е $з$ ю м е

У моделі ефективних мас та прямокутних потенціалів із залученням модифікованого варіаційного методу Бете досліджено спектральні параметри електрона в багатошаровій циліндричній напівпровідниковій нанотрубці з донорною домішкою на аксіальній осі. Проаналізовано залежності енергій зв'язку електрона з домішкою та сил осциляторів внутрішньозонних оптичних квантових переходів від геометричних параметрів складної нанотрубки на основі напівпровідників GaAs та $\mathrm{Al}_{0,4} \mathrm{Ga}_{0,6} \mathrm{As}$. 